\title{
Impact of Energy Slope Averaging Methods on Numerical Solution of 1D Steady Gradually Varied Flow
}

\author{
Wojciech Artichowicz, Dzmitry Prybytak \\ Gdańsk University of Technology, Faculty of Civil and Environmental Engineering, \\ ul. G. Narutowicza 11/12, 80-233 Gdańsk, Poland, e-mail: wojartic@pg.gda.pl
}

(Received October 09, 2015; revised December 03, 2015)

\begin{abstract}
In this paper, energy slope averaging in the one-dimensional steady gradually varied flow model is considered. For this purpose, different methods of averaging the energy slope between cross-sections are used. The most popular are arithmetic, geometric, harmonic and hydraulic means. However, from the formal viewpoint, the application of different averaging formulas results in different numerical integration formulas. This study examines the basic properties of numerical methods resulting from different types of averaging.
\end{abstract}

Key words: standard step method, numerical integration, initial value problem, ordinary differential equation, open channel, steady gradually varied flow, energy slope

\begin{tabular}{|c|c|}
\hline \multicolumn{2}{|r|}{ List of symbols } \\
\hline$A$ & - wetted cross-sectional area; \\
\hline$c$ & - any constant in the stability analysis problem; \\
\hline$f(x, y)=y^{\prime}(x)$ & - derivative of the function $y(x)$ \\
\hline$g$ & - gravitational acceleration; \\
\hline$g_{(\delta)}$ & - function interpolating the integrand; \\
\hline$h$ & - water level elevation above the assumed datum; \\
\hline$L$ & - length of the considered channel reach; \\
\hline$n$ & _ Manning's roughness coefficient; \\
\hline$Q$ & - flow discharge; \\
\hline$\widetilde{R}$ & - hydraulic radius; \\
\hline$S$ & - energy slope; \\
\hline$\overline{S_{i}}$ & $\begin{array}{l}\text { - average slope of the energy line in the channel reach } \\
\text { bounded by the cross-sections } i \text { and } i+1 \text {; }\end{array}$ \\
\hline$U$ & - average velocity in a cross section; \\
\hline$x$ & $\begin{array}{l}\text { - spatial variable in the energy equation or the independent } \\
\text { variable in the ordinary differential equation; }\end{array}$ \\
\hline
\end{tabular}




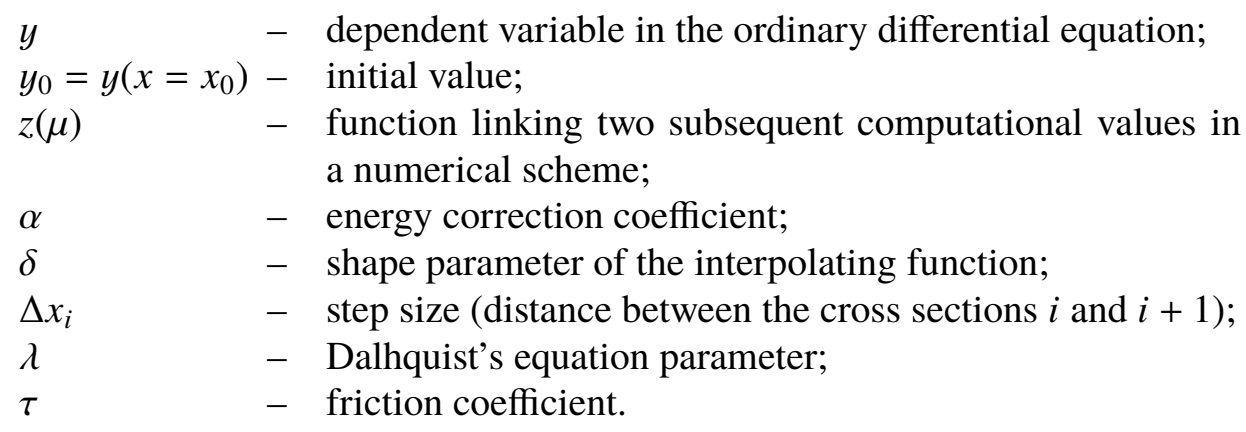

\section{Introduction}

One-dimensional steady gradually varied flow (SGVF) in open channels is a basic subject of interest for hydraulic engineers. The usual approach to analysing SGVF, commonly called the standard step method (Chanson 2004, Chow 1959, French 1985), is a state-of-the-art methodology for finding the flow profile along the channel. This method can be interpreted as a result of the application of the discrete Bernoulli's equation to a channel reach.

For a channel reach bounded by neighbouring cross-sections indexed $i$ and $i+1$ (Fig. 1), Bernoulli's equation has the following form:

$$
h_{i+1}+\alpha_{i+1} \frac{U_{i+1}^{2}}{2 g}=h_{i}+\alpha_{i} \frac{U_{i}^{2}}{2 g}-\Delta x_{i} \cdot \overline{S_{i}},
$$

where:

$\Delta x_{i} \quad-\quad$ step size (distance between the cross sections $i$ and $i+1$ ),

$U$ - average velocity in a cross section,

$h$ - water surface elevation above the assumed datum,

A - wetted cross-sectional area,

$g-$ gravitational acceleration,

$\frac{\alpha}{S_{i}}-$ energy correction coefficient,

$\overline{S_{i}} \quad-$ average slope of the energy line in the channel reach bounded by the cross-sections $i$ and $i+1$.

Graphical interpretation of Eq. 1 is displayed in Fig. 1.

To estimate the energy slope $\overline{S_{i}}$, the arithmetic mean of friction slopes in crosssections involved is usually used (Chanson 2004, Chow 1959, French 1985):

$$
\overline{S_{i}}=\frac{1}{2}\left(S_{i}+S_{i+1}\right) \text {. }
$$

The friction slope in a cross-section can be computed by Manning's formula (French 1985):

$$
S=\frac{Q^{2} \cdot n^{2}}{R^{4 / 3} \cdot A^{2}}
$$




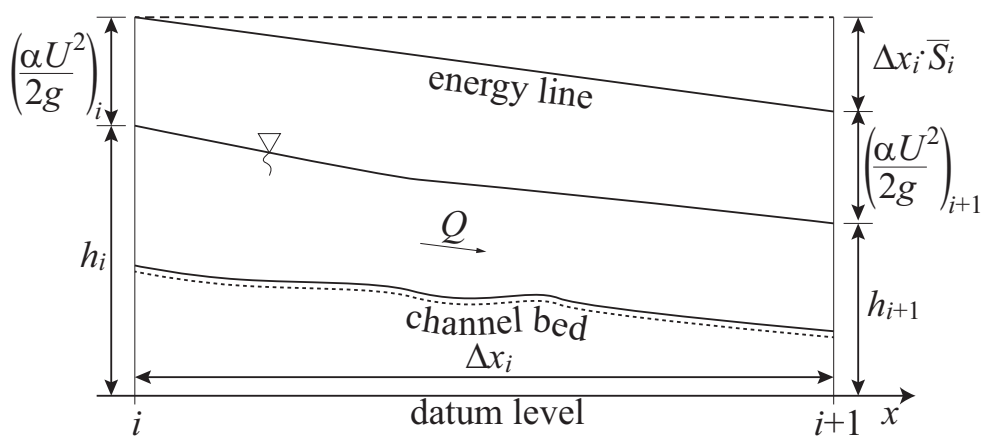

Fig. 1. Graphical interpretation of Eq. (1)

where $R$ denotes hydraulic radius, and $n$ is Manning's roughness coefficient. Alternatively, the energy slope can be estimated by the Darcy-Weisbach formula (Chanson 2004):

$$
S=\frac{Q^{2} \cdot \tau}{8 \cdot g \cdot R \cdot A^{2}}
$$

where $\tau$ is a friction coefficient.

To increase the accuracy of computations, some authors introduce different types of energy slope averaging, which are supposed to perform better with different types of flow profiles. The most popular ones are - the hydraulic mean

$$
\bar{S}=\left(\frac{Q_{i}+Q_{i+1}}{K_{i}+K_{i+1}}\right)^{2}
$$

in which $Q$ is the flow rate, and $K$ denotes conveyance. If $Q=$ const., Eq. 5 yields

$$
\bar{S}=\left(\frac{2 Q}{K_{i}+K_{i+1}}\right)^{2},
$$

- the geometric mean

$$
\bar{S}=\sqrt{S_{i} \cdot S_{i+1}},
$$

- the harmonic mean

$$
\bar{S}=\frac{2 \cdot S_{i} \cdot S_{i+1}}{S_{i}+S_{i+1}} .
$$

HEC-RAS Hydraulic Reference Manual (US Army Corps of Engineers 2010) lists the above formulas along with the flow profiles for which they should assure the best accuracy. Apart from these formulas, some researchers propose their own (Reed and Wolfkill 1976, Laurenson 1986, Chadderton and Miller 1980).

Except for arithmetic mean averaging, the resulting formulas have not been formally examined. Chadderton and Miller (1980) analysed slope averaging formulas in means for numerical error and accuracy by performing a set of numerical tests. 
However, they based their research on numerical experiments without comparing the results with analytical solutions. Laurenson's (1986) analysis was based on an arbitrarily assumed slope friction function, which was not an analytical solution of the flow equation. Artichowicz and Mikos-Studnicka (2014) compared the numerical solutions of the one-dimensional energy flow equation with analytical solutions, and concluded that differences between the outcomes of formulas 2, 5, 6, 7 and 8 are insignificant from the practical viewpoint. None of the researchers presents detailed discussion of the origin of those methods or the consequences and formalism of their usage. The introduction of different formulas for slope averaging is treated as a simple substitution of one mean for another, and it is done without any comment on the formalism of these proceedings. Such substitutions are often found in hydraulics, not only in one-dimensional SGVF modelling in open channels, but also in two-dimensional flood modelling with a diffusive wave (Gąsiorowski 2013). However, switching to another averaging method has serious numerical consequences, as it is equivalent to using a different type of the numerical integration scheme of the ordinary differential equation (ODE). If so, its numerical properties, such as stability, consistence and convergence, may also differ. To the best of the authors' knowledge, there is no thorough analysis of the above-mentioned methods using averaging formulas other than the arithmetic mean.

In the following sections, it will be shown that the use of formulas 2, 7 and 8 for energy slope averaging in the standard step method is in fact the application of the one-step generalized integration rule (GIR) with respect to a function interpolating the integrand in which a certain value of the shape parameter is used. Numerical properties of GIRs with geometric and harmonic mean averaging will be examined. The application of formula 5 yields another integration scheme as well, but it is not an instance of the family of GIRs considered here and will not be discussed in this paper.

\section{The Governing Equation of SGVF}

The governing equation of SGVF can be derived from the system of Saint-Venant equations (Artichowicz and Szymkiewicz 2014). In the case of flow with discharge constant along the channel reach, the flow equation takes the following form:

$$
\frac{d}{d x}\left(h+\frac{\alpha \cdot Q^{2}}{2 g \cdot A^{2}}\right)=-S,
$$

where $x$ is the spatial variable. The ordinary differential equation obtained, which represents the energy principle of one-dimensional open channel flow can be written in its shorter form

$$
\frac{d E}{d x}=-S
$$


with

$$
E=h+\frac{\alpha \cdot Q^{2}}{2 g \cdot A^{2}} .
$$

Eq. 10 is one of the possible forms of the governing equation of SGVF. To solve it, the initial value problem (IVP) has to be stated (Ascher and Petzold 1998, Kincaid and Cheney 2002). In the case considered here, this means that the energy stage has to be imposed on one of the bounds of the solution domain (channel reach) (Szymkiewicz 2010). If flow is subcritical, the energy stage should be imposed at the outflow cross-section, so the initial condition will take the following form:

$$
E_{L}=E\left(x_{L}=L\right)
$$

with $L$ denoting the length of the channel reach. In the case of supercritical flow, the energy stage is imposed at the inflow cross-section, and the initial value becomes:

$$
E_{0}=E\left(x_{0}=0\right) \text {. }
$$

In practice, this means that to solve the IVP for Eq. 10, water surface elevation at the first or last cross-section of the channel reach has to be known, as well as the flow rate $Q$.

\section{Standard Step Method as an Application of the Trapezoidal Rule to SGVF}

Consider the initial value problem for the general ordinary differential equation (Ascher and Petzold 1998):

$$
\frac{d y(x)}{d x}=f(x, y(x))
$$

with

$$
y_{0}=y\left(x=x_{0}\right)
$$

where

$$
\begin{array}{ll}
x & - \text { independent variable, } \\
y=y(x) & - \text { dependent variable, } \\
f(x, y)=y^{\prime}(x) & - \text { derivative of the function } y(x), \\
y_{0}=y\left(x=x_{0}\right) & - \text { initial value. }
\end{array}
$$

If the problem stated by Eqs. 14 and 15 cannot be solved analytically, numerical methods have to be applied. There are many such methods: for example, the Euler backward (implicit) and forward (explicit) methods, the family of Runge-Kutta methods or the trapezoidal rule. Their detailed descriptions, advantages and disadvantages can be found, for example, in Ascher and Petzold (1998), Kincaid and Cheney (2002) and many other publications devoted to numerical methods. The applications 
of such methods to hydro-engineering problems are given, among others, by Cunge et al (1979) and Szymkiewicz (2010).

One of the methods most frequently used in open channel hydraulics because of its excellent numerical properties is the trapezoidal rule (Szymkiewicz 2010, Ascher and Petzold 1998):

$$
y_{i+1}=y_{i}+\frac{\Delta x_{i}}{2} \cdot\left[f\left(x_{i}, y_{i}\right)+f\left(x_{i+1}, y_{i+1}\right)\right] .
$$

The trapezoidal rule can be derived in many ways - for example, by expanding the Taylor series. Step-by-step derivation can be found in Ascher and Petzold (1998).

The application of the trapezoidal rule to energy Eq. 10 yields its discrete form:

$$
E_{i+1}=E_{i}-\frac{\Delta x_{i}}{2} \cdot\left(S_{i}+S_{i+1}\right) \text {. }
$$

After the introduction of the average flow velocity $U=Q / A$ and Eq. 11, a given form of the standard step method is obtained (Chow 1959, Cunge et al 1979, French 1985, Szymkiewicz 2010, US Army Corps of Engineers 2010):

$$
h_{i+1}+\alpha_{i+1} \frac{U_{i+1}^{2}}{2 g}=h_{i}+\alpha_{i} \frac{U_{i}^{2}}{2 g}-\frac{\Delta x_{i}}{2} \cdot\left(S_{i}+S_{i+1}\right) .
$$

The algebraic non-linear equation obtained has only one unknown, depending on the required direction of integration: $h_{i}$ if flow is subcritical, or $h_{i+1}$ if it is supercritical. All other variables are known from the initial condition or the previous step of computation. Solving this equation iteratively in subsequent cross-sections, makes it possible to obtain the searched-for water stage profile along the channel.

It can be noticed that the standard step method is in fact a numerical approximation of the energy equation by the trapezoidal rule, in which the average slope is estimated with the arithmetic mean.

\section{Integration Rule Generalized with Respect to the Function Interpolating the Integrand}

The trapezoidal rule can be derived by a different method than the one presented in the previous section. To solve the IVP, the subsequent approximations $y_{i+1}$ of the unknown function $y\left(x_{i+1}\right)$ can be found, starting from the initial value and evaluating an integral. Knowing $y_{i}$ from the initial value $y_{0}=y\left(x=x_{0}\right)$ or from the previous step of computations, it is possible to write that (LeVeque 2007)

$$
y_{i+1}=y_{i}+\int_{x_{i}}^{x_{i+1}} f(x, y) d x .
$$

If the integral in Eq. 19 cannot be solved analytically, it has to be approximated numerically. Generally, any quadratures can be used for this purpose (Kincaid and Cheney 
2002). However Gaussian quadratures and similar approaches require function values between $x_{i}$ and $x_{i+1}$, and therefore are not valid for open channel computations, as channel parameters are known in computational nodes only. For this reason, rectangular (backward or forward Euler) or trapezoidal methods are often used, as they require the knowledge of only cross-sectional parameters in computational nodes. The process of numerical integration can be interpreted as the integration of a function $g(x, y)$ interpolating the original $f(x, y)$ function. In other words, $f(x, y)$ is replaced with $g(x, y)$, which is usually easy to integrate analytically. Typical examples of such functions are piecewise constant, linear or polynomial functions. However, any function $g(x, y)$ can be used as the interpolating one. Formalizing the above observations, it can be written that

$$
\int_{x_{i}}^{x_{i+1}} f(x, y) d x \approx \int_{x_{i}}^{x_{i+1}} g(x, y) d x .
$$

In the case of the forward Euler method, the piecewise constant interpolation of the function $f(x, y)$ is performed. An example of such integration is shown in Fig. 2a. In the case when a linear function is used, the trapezoidal rule with arithmetic mean averaging is obtained (Fig. 2b).

a)

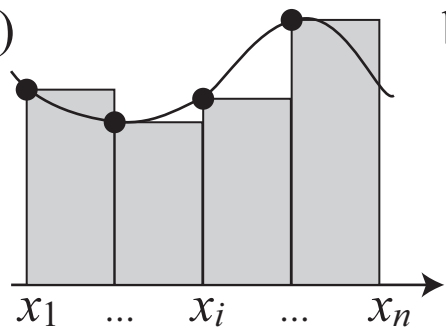

b)

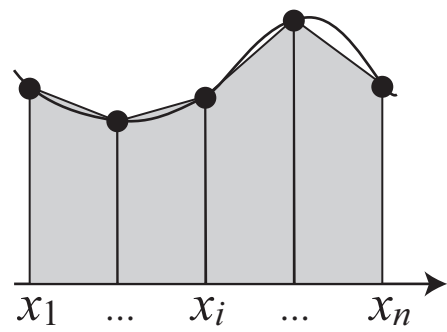

Fig. 2. Graphical interpretation of numerical methods for solving the IVP:

a) forward Euler method, b) trapezoidal rule.

Let us introduce the following notation: $f_{i}=f\left(x_{i}, y\left(x_{i}\right)\right)$ and $f_{i+1}=f\left(x_{i+1}, y\left(x_{i+1}\right)\right)$. Then the function $g_{(1)}(x, y)$, which interpolates linearly $f_{i}$ and $f_{i+1}$, is expressed as:

$$
g_{(1)}(x, y)=\frac{f_{i+1}-f_{i}}{x_{i+1}-x_{i}}\left(x-x_{i}\right)+f_{i},
$$

which after integration yields:

$$
\int_{x_{i}}^{x_{i+1}} g_{(1)}(x, y)=\frac{\left(x_{i+1}-x_{i}\right)}{2}\left(f_{i}+f_{i+1}\right)=\frac{\Delta x_{i}}{2}\left(f_{i}+f_{i+1}\right) .
$$

Substitution of Eq. 22 into Eq. 19 gives the well-known trapezoidal rule:

$$
y_{i+1}=y_{i}+\frac{\Delta x_{i}}{2} \cdot\left(f_{i}+f_{i+1}\right) \text {. }
$$


Let us consider a general interpolating function

$$
g_{(\delta)}(x, y)=\left(\frac{\left(f_{i+1}\right)^{\delta}-\left(f_{i}\right)^{\delta}}{x_{i+1}-x_{i}}\left(x-x_{i}\right)+\left(f_{i}\right)^{\delta}\right)^{\frac{1}{\delta}},
$$

in which $\delta$ is a real-valued parameter influencing the type of interpolation between points $\left(x_{i}, f_{i}\right)$ and $\left(x_{i+1}, f_{i+1}\right)$. Hence, when linear interpolation is used, the value $\delta=1$ yields Eq. 23. When Eq. 24 with $\delta=-1 / 2$ is applied, the interpolating function takes the following form:

$$
g_{\left(-\frac{1}{2}\right)}(x, y)=\left(\frac{\left(f_{i+1}\right)^{-1 / 2}-\left(f_{i}\right)^{-1 / 2}}{x_{i+1}-x_{i}}\left(x-x_{i}\right)+\left(f_{i}\right)^{-1 / 2}\right)^{-2} .
$$

Integration of Eq. 25 yields:

$$
\int_{x_{i}}^{x_{i+1}} g_{\left(-\frac{1}{2}\right)}(x, y)=\left(x_{i+1}-x_{i}\right) \cdot \sqrt{f_{i} \cdot f_{i+1}}=\Delta x_{i} \cdot \sqrt{f_{i} \cdot f_{i+1}} .
$$

Introduction of Eq. 26 into Eq. 19 results in another numerical integration scheme, which is an instance of the GIR:

$$
y_{i+1}=y_{i}+\Delta x_{i} \cdot \sqrt{f_{i} \cdot f_{i+1}} .
$$

It can be noticed that the geometric mean of the derivatives of $y(x)$ in computational nodes is obtained, and it can be used only if $f_{i}$ and $f_{i+1}$ are greater than zero.

After repeating these steps with $\delta=-2$, we obtain another form of the interpolating function:

$$
\begin{gathered}
g_{(-2)}(x, y)=\left(\frac{\left(f_{i+1}\right)^{-2}-\left(f_{i}\right)^{-2}}{x_{i+1}-x_{i}}\left(x-x_{i}\right)+\left(f_{i}\right)^{-2}\right)^{-1 / 2}, \\
\int_{x_{i}}^{x_{i+1}} g_{(-2)}(x, y)=\left(x_{i+1}-x_{i}\right) \cdot \frac{2 f_{i} \cdot f_{i+1}}{f_{i}+f_{i+1}}=\Delta x_{i} \cdot \frac{2 f_{i} \cdot f_{i+1}}{f_{i}+f_{i+1}},
\end{gathered}
$$

resulting in another method for solving the IVP:

$$
y_{i+1}=y_{i}+\Delta x_{i} \cdot \frac{2 f_{i} \cdot f_{i+1}}{f_{i}+f_{i+1}} .
$$

In Eq. 30, the harmonic mean of the derivatives of $y(x)$ in computational nodes is obtained. Formulas 27 and 30 are nonlinear integration methods and instances of the GIR. For comparison, to highlight the differences between the formulas obtained, functions $g_{(1)}, g_{(-1 / 2)}, g_{(-2)}$ were applied to interpolate between two arbitrarily chosen points $(0,1)$ and $(2,27)$. The result is shown in Fig. 3. 


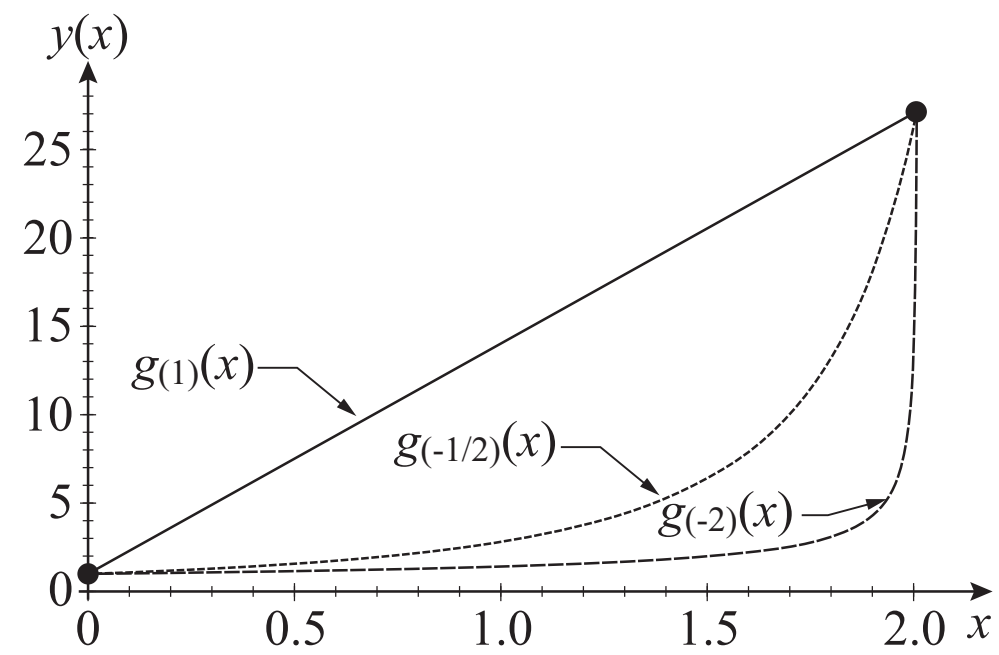

Fig. 3. Example of interpolation between points $(0,1)$ and $(2,27)$ with $g_{(1)}, g_{(-1 / 2)}, g_{(-2)}$.

As it can be noticed, the functions $g_{(1)}, g_{(-1 / 2)}, g_{(-2)}$ have different shapes. The function $g_{(1)}$ interpolates linearly, whereas $g_{(-1 / 2)}$ and $g_{(-2)}$ interpolate with hyperbolic functions. The graphical interpretation of the integration of the IVP with formulas 27 and 30 are depicted in Fig. 4.
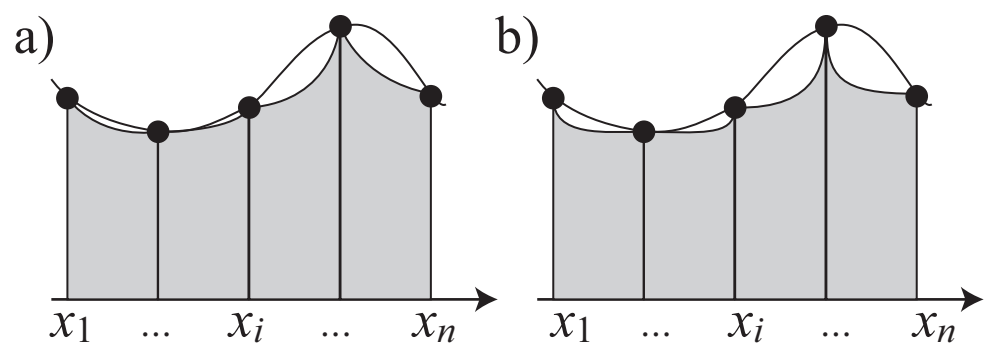

Fig. 4. Graphical interpretation of integration with functions a) $g_{(-1 / 2)}$ and b) $g_{(-2)}$.

\section{Solution of the IVP for Dahlquist's Test Equation}

As shown in the previous section, the introduction of different energy slope averaging methods is in fact the application of different numerical methods for the integration of the ODE. Such methods differ in their numerical properties, which influence the solution obtained. The most important properties are stability, consistency and convergence.

The properties are usually obtained by examining the behaviour of the method applied to so-called test equations (Ascher and Petzold 1998, Gustafsson 2011, Hairer 
and Wanner 2010). To study stability, Dalhquist's test equation is used:

$$
\frac{d y}{d x}=\lambda \cdot y
$$

with

$$
y\left(x_{0}=0\right)=y_{0},
$$

where $\lambda$ is a complex number. It is necessary to apply the numerical method to a stable IVP. The IVP considered here is stable if the real part of $\lambda$ is less than zero: $\mathfrak{R}(\lambda) \leq$ 0 . In such a case, the perturbations of the numerical solution decay with increasing values of the independent variable $x(x \geq 0)$. A detailed discussion of IVP stability according to the $\lambda$ parameter can be found in Ascher and Petzold (1998). In the present case, the IVP has an exact solution:

$$
y(x)=y_{0} \cdot e^{\lambda \cdot x} .
$$

To examine the stability properties of the numerical integration formula, it is necessary to apply the formula to the test Eq. 31 and to express it in a general form linking the previous and following computational steps with a function denoted as $z(\mu)$ :

$$
y_{i+1}=z(\mu) \cdot y_{i},
$$

where $\mu=\lambda \cdot \Delta x$. The integration step $\Delta x=\Delta x_{i}=x_{i+1}-x_{i}$ is assumed to be constant. The $z(\mu)$ function is obtained by rearrangement of the numerical method formula. The application of the trapezoidal method with arithmetic averaging $(\delta=1)$ (Eq. 23) to the test Eq. 31 yields

$$
y_{i+1}=y_{i}+\frac{\lambda \cdot \Delta x}{2} \cdot\left(y_{i}+y_{i+1}\right) .
$$

After rearranging Eq. 35 to the general form, one obtains

$$
y_{i+1}=\frac{1+\lambda \cdot \frac{\Delta x}{2}}{1-\lambda \cdot \frac{\Delta x}{2}} \cdot y_{i},
$$

so the $z(\mu)$ function for the trapezoidal rule with arithmetic mean averaging has the following form:

$$
z_{(1)}(\mu)=\frac{1+\frac{\mu}{2}}{1-\frac{\mu}{2}} .
$$

The value in the subscript denotes the interpolation parameter $\delta$ introduced in the previous section (here $\delta=1$ ).

The application of the integration rule with geometric $(\delta=-1 / 2)$ and harmonic averaging $(\delta=-2)$ to the test Eq. 31 yields respectively:

$$
y_{i+1}=y_{i}+\lambda \cdot \Delta x \sqrt{y_{i} \cdot y_{i+1}},
$$




$$
y_{i+1}=y_{i}+2 \lambda \cdot \Delta x \frac{y_{i} \cdot y_{i+1}}{y_{i}+y_{i+1}} .
$$

The formulas obtained are implicit and non-linear with respect to $y_{i+1}$, and it is impossible to express them in a general form of Eq. 34. For this reason they can be treated as non-linear equations with the unknown variable $y_{i+1}$. Solving each of them with respect to this variable, one obtains different forms of these formulas. Eq. 38 is then rearranged into

$$
y_{i+1}=\frac{1}{2}\left(2+(\lambda \cdot \Delta x)^{2} \pm \sqrt{4(\lambda \cdot \Delta x)^{2}+(\lambda \cdot \Delta x)^{4}}\right) \cdot y_{i}
$$

whereas Eq. 39 is rearranged into

$$
y_{i+1}=\left(\lambda \cdot \Delta x \pm \sqrt{1+(\lambda \cdot \Delta x)^{2}}\right) \cdot y_{i} .
$$

This makes it possible to express geometrically and harmonically averaged integration methods in a general form given by Eq. 34. In both cases, there are two possible expressions obtained, which differ in the sign before the square root term. Formula 40 is an exact equivalent of numerical integration formula 38 if the sign before the square root term is chosen on the basis of the sign of the real part of $\lambda$ :

$$
z_{(-1 / 2)}(\mu)=\frac{1}{2}\left(2+\mu^{2}+\operatorname{sgn}(\mathfrak{R}(\mu)) \cdot \sqrt{4 \mu^{2}+\mu^{4}}\right) .
$$

Formula 41 is an exact equivalent of formula 39 if, irrespective of the value of $\lambda$, the expression with the plus sign preceding the square root is taken. Thus the $z(\mu)$ function for the GIR with harmonic averaging is given by

$$
z_{(-2)}(\mu)=\mu+\sqrt{1+\mu^{2}} .
$$

\section{Stability Analysis}

The numerical method for the IVP is absolutely stable if

$$
|z(m)|<1
$$

when a stable IVP for test Eq. 31 is considered. This means that, when the numerical approximation scheme is applied to Dahlquist's test equation, any two subsequent values fulfil the following condition (Ascher and Petzold 1998, Gustafsson 2011):

$$
\left|y_{i+1}\right| \leq\left|y_{i}\right|, \text { for } \mathrm{i}=0,1, \ldots
$$

The trapezoidal rule given with Eq. 23 is very popular and thus well examined. It is consistent, 0-stable, absolutely stable, A-stable and therefore convergent (Ascher and Petzold 1998). 
In the present test case, the computational step is assumed to be greater than zero $(\Delta x>0)$, and the test equation parameter is less than zero $(\lambda=-1)$, so the value $\mu=\lambda \cdot \Delta x$ is always less than zero. The plots of the functions $\left|z_{(1)}(\mu)\right|,\left|z_{(-1 / 2)}(\mu)\right|$ and $\left|z_{(-2)}(\mu)\right|$ are depicted in Fig. 5. It can be noticed that both $\left|z_{(-1 / 2)}(\mu)\right|$ and $\left|z_{(-2)}(\mu)\right|$ decrease towards minus infinity, whereas for $\mu=0$ they reach a value of 1 . This suggests that both functions fulfil the stability condition expressed by inequality 44 .

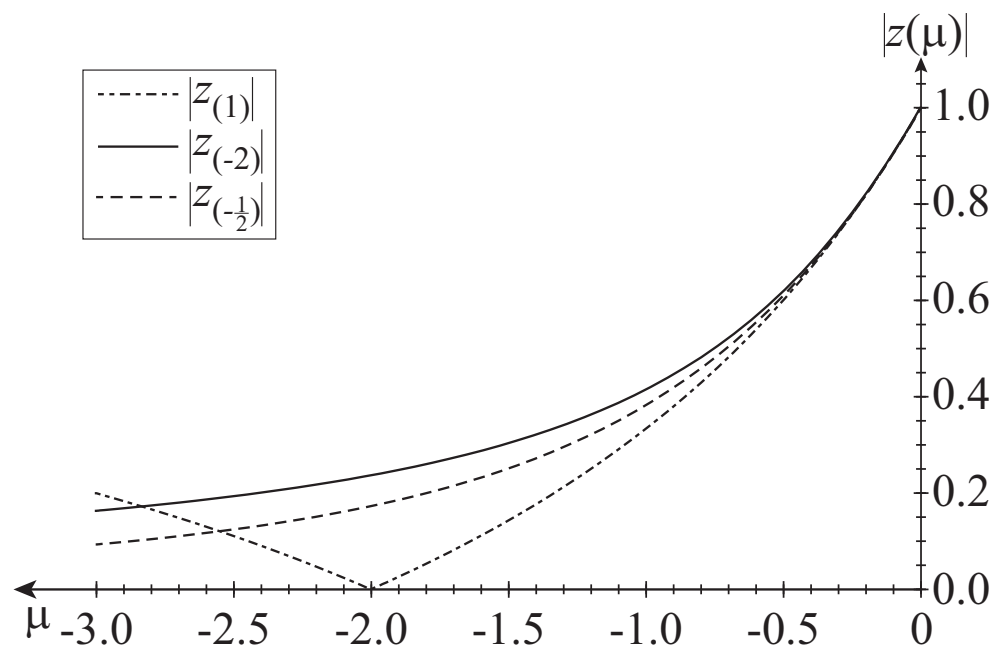

Fig. 5. Plot of the functions $z_{(1)}(\mu), z_{(-2)}(\mu)$ and $z_{(-1 / 2)}(\mu)$.

To show this explicitly, let us take two values: $\mu_{1}>\mu_{2}$ (note that $\mu_{1}, \mu_{2} \leq 0$ ). To demonstrate that the functions $z_{(-1 / 2)}(\mu)$ and $z_{(-2)}(\mu)$ always fulfil condition 44 , it is enough to show that they decrease as $\mu$ goes to minus infinity. These functions reach a value of 1 , which is to be a maximum, for $\mu=0$. The function $z_{(-1 / 2)}(\mu)$ can be rearranged to

$$
\begin{aligned}
z_{\left(-\frac{1}{2}\right)}(\mu) & =\frac{1}{2}\left(2+\mu^{2}-\sqrt{4 \mu^{2}+\mu^{4}}\right) \frac{\left(2+\mu^{2}+\sqrt{4 \mu^{2}+\mu^{4}}\right)}{2+\mu^{2}+\sqrt{4 \mu^{2}+\mu^{4}}}= \\
& =\frac{2}{2+\mu^{2}+\sqrt{4 \mu^{2}+\mu^{4}}}
\end{aligned}
$$

whereas the function $z_{(-2)}(\mu)$ can be expressed as

$$
z_{(-2)}(\mu)=\left(\mu+\sqrt{1+\mu^{2}}\right) \frac{\mu-\sqrt{1+\mu^{2}}}{\mu-\sqrt{1+\mu^{2}}}=\frac{1}{\sqrt{1+\mu^{2}}-\mu} .
$$

In both cases the two functions are the inverses of expressions which increase as $\mu$ goes to minus infinity. Therefore $z_{(-1 / 2)}\left(\mu_{1}\right)>z_{(-1 / 2)}\left(\mu_{2}\right)$ and $z_{(2)}\left(\mu_{1}\right)>z_{(2)}\left(\mu_{2}\right)$, which 
proves that these functions are monotonic and therefore can never exceed a value of 1 for $\mu \in(-\infty, 0]$. Thus the integration rule with geometric or harmonic averaging fulfils condition 44 , which means that both methods are absolutely stable.

The numerical method is A-stable if its region of absolute stability (Eq. 44) covers the complex negative half-plane of $\mu$. The trapezoidal rule is proved to be an A-stable method (Ascher and Petzold 1998), and the set of $\mu$ values for which the absolute stability condition is fulfilled is depicted in Fig. 6a. When harmonic averaging is used, the integration method is A-stable as well and has the same set of values that fulfil the stability condition as the trapezoidal rule (Fig. 6a). However, when geometric averaging is used, the integration method is not A-stable, as its region of absolute stability does not cover the whole complex left half-plane (Fig. 6b).

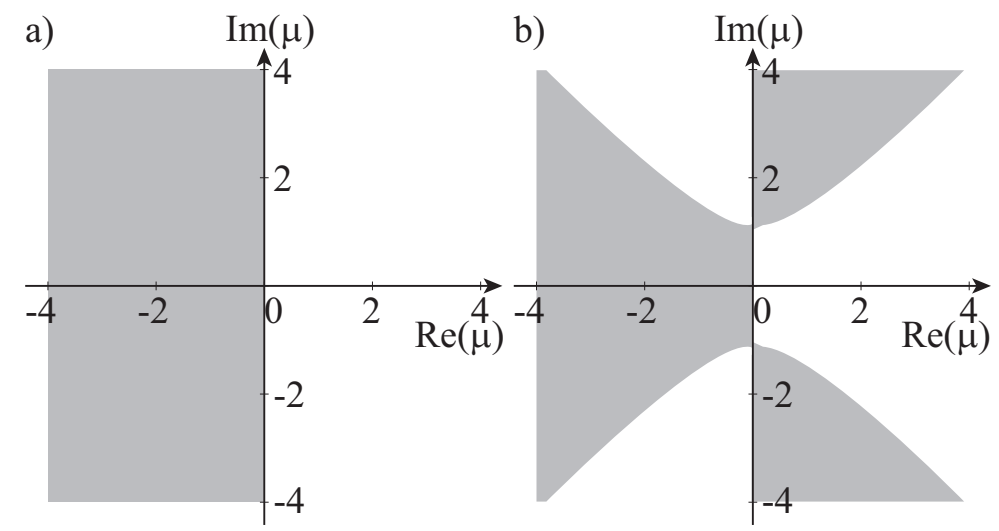

Fig. 6. Plot of the regions where a) $\left|z_{(1)}(m)\right| \leq 1$ and $\left|z_{(-1 / 2)}(m)\right| \leq 1$, b) $\left|z_{(-2)}(m)\right| \leq 1$.

Another type of stability is 0 -stability, which depends on the behaviour of the numerical method in terms of round-off errors. A practical way to test the zero stability property is to use the method to solve the ODE which has a constant solution. An example test problem can be the following IVP (LeVeque 2007):

$$
\frac{d y}{d x}=0
$$

with

$$
y\left(x_{0}=0\right)=c,
$$

where $c$ is a constant value. If a numerical one-step method is 0 -stable, when applied to (48), it results in the formula

$$
y_{i+1}=y_{i}
$$


as $f(x, y)=0$ for any $x$ and $y$. When the GIR with arithmetic (Eq. 23) or geometric (Eq. 27) averaging is applied to Eq. 48, the resulting formula is Eq. 50, so these methods are 0 -stable. However, when the GIR with harmonic averaging (Eq. 30) is applied to Eq. 48 , an indeterminate expression is obtained:

$$
y_{i+1}=y_{i}+\Delta x_{i} \cdot \frac{2 f_{i} \cdot f_{i+1}}{f_{i}+f_{i+1}}=y_{i}+\Delta x_{i} \cdot \frac{0}{0},
$$

so this method is not 0 -stable.

\section{Consistency Analysis}

To test the consistency of the method, let us assume that the function $y(x)$ is known. Expressing the value $y_{i+1}$ in terms of the Taylor expansion at point $x_{i}$, one obtains

$$
y_{i+1}=\sum_{k=0}^{\infty} \frac{\Delta x^{k}}{k !} \cdot y_{i}^{(k)} \text {. }
$$

To find the order of the method, all the terms are moved to one side of the equation, and the Taylor expansion (Eq. 52) is introduced instead of $y_{i+1}$. The term with the lowest power of the integration step of the remaining part denotes the method's order of consistency. For the trapezoidal rule, the following expression is obtained:

$$
e_{(1)}=\frac{y_{i+1}-y_{i}}{\Delta x}-\frac{1}{2}\left(y_{i}^{\prime}+y_{i+1}^{\prime}\right)=-\frac{\Delta x^{2}}{12} y_{i}^{\prime \prime \prime}+O\left(\Delta x^{3}\right) \text {. }
$$

In the case of the methods with geometric and harmonic averaging, manipulations of Taylor's series have to be applied to obtain the result. For the integration rule with geometric averaging, the following formula is obtained:

$$
e_{\left(-\frac{1}{2}\right)}=\frac{y_{i+1}-y_{i}}{\Delta x}-\sqrt{y_{i}^{\prime} \cdot y_{i+1}^{\prime}}=\Delta x^{2} \cdot\left(\frac{1}{6} y_{i}^{\prime \prime \prime}-\frac{1}{2}\left(\frac{y_{i}^{\prime \prime \prime}}{2}-\frac{y_{i}^{\prime \prime 2}}{4 y_{i}^{\prime}}\right)\right)+O\left(\Delta x^{3}\right),
$$

whereas the rule with harmonic averaging yields

$$
\begin{gathered}
e_{(-2)}=\frac{y_{i+1}-y_{i}}{\Delta x}-\frac{2 y_{i}^{\prime} \cdot y_{i+1}^{\prime}}{y_{i}^{\prime}+y_{i+1}^{\prime}}= \\
=\Delta x^{2} \cdot\left\{\frac{1}{6} y_{i}^{\prime \prime \prime}-2 y_{i}^{\prime} \cdot\left[\frac{1}{4}\left(\frac{y_{i}^{\prime \prime \prime}}{y_{i}^{\prime}}-\frac{y^{\prime \prime 2}}{y_{i}^{\prime 2}}\right)+\frac{1}{8}\left(\frac{y_{i}^{\prime \prime}}{y_{i}^{\prime 2}}-\frac{y^{\prime \prime \prime}{ }_{i}}{y_{i}^{\prime}}\right)\right]\right\}+O\left(\Delta x^{3}\right) .
\end{gathered}
$$

This shows that each integration method is consistent of the second order.

\section{Convergence}

Convergence is obtained for every method which is 0 -stable and consistent. Among the methods examined, only the trapezoidal rule and the GIR with geometric averaging are convergent, as they fulfil both conditions. When the harmonic mean is used for averaging, the method is not $0-$ stable and therefore not convergent. 


\section{Impact of the Slope Averaging Method and Manning's Roughness Coefficient on the Solution of the Flow Equation}

Different integration methods are applied to the SGVF equation in order to increase the accuracy of its numerical solution. It seems, however, that the greatest error is produced by an inaccurate estimation of Manning's roughness coefficient, whereas errors generated by the choice of the numerical solution method are irrelevant.

To examine the impact of the numerical method and Manning's roughness coefficient, a test case was formulated by means of the analytic benchmark solution of 1D SGVF (MacDonald et al 1997), with which numerical outcomes were compared. The benchmark solution is constructed by formulating the inverse problem for the SGVF equation, in which the bed level function $z(x)$ is estimated for an assumed depth function $H(x)$. Apart from the depth function, the data required to find the channel bed level function, are flow discharge and channel geometry: channel width $B(x, H(x))$, active flow area $A(x, H(x))$ and wetted perimeter $P(x, H(x))$. The energy slope function $S(x, H(x))$ is estimated by formula 3 . The bed slope function is given by

$$
s_{b}(x)=\frac{d H}{d x}+S(x, H(x))+\frac{\alpha \cdot Q^{2}}{2 g} \frac{d}{d x}\left(\frac{1}{A^{2}(x, H(x))}\right) .
$$

The bed level corresponding to the imposed depth function $H(x)$ and channel geometry can be found by integrating the bed slope function 56 :

$$
z(x)=-\int_{x}^{L} s_{b}(\chi) d \chi .
$$

The benchmark solution was generated for a prismatic channel with the following properties: constant channel width $b=5 \mathrm{~m}$, channel length $L=1000 \mathrm{~m}$, Manning's roughness coefficient $n=0.03 \mathrm{~s} / \mathrm{m}^{1 / 3}$, flow discharge $Q=5 \mathrm{~m}^{3} / \mathrm{s}$, energy correction coefficient $\alpha=1.1$. The gravitational acceleration was assumed $g=9.81 \mathrm{~m} / \mathrm{s}^{2}$. The assumed depth function is

$$
H(x)=\frac{9}{8}+\frac{1}{4} \sin \left(\frac{\pi \cdot x}{500}\right) .
$$

The channel bed function $z(x)$ and the water surface level $h(x)=z(x)+H(x)$ as well as the energy line and the critical depth level obtained for this flow case are depicted in Fig. 7.

Numerical computations were performed by integration methods using different types of energy slope averaging: arithmetic, geometric and harmonic means. Two solution cases were considered. In the first case, the computations were performed for the data used to construct the benchmark solution. In the second case, the roughness 


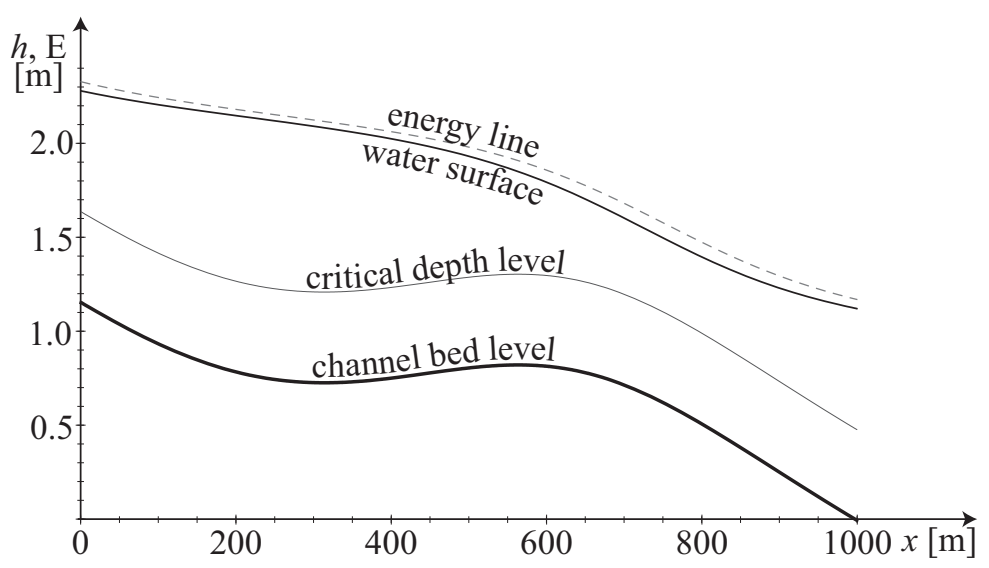

Fig. 7. Generated analytic benchmark solution.

coefficient was changed from $n=0.03 \mathrm{~s} / \mathrm{m}^{1 / 3}$ to $n=0.031 \mathrm{~s} / \mathrm{m}^{1 / 3}$ to highlight the impact of this parameter on the numerical solution. The integration step assumed was $\Delta x=50 \mathrm{~m}$.

To examine the quality of numerical solutions, the mean square error was computed

$$
M S E=\frac{1}{N} \sum_{i}\left(H_{i}-H\left(x_{i}\right)\right)^{2},
$$

where $H_{i}$ denotes depth values obtained from the numerical solution, whereas $H\left(x_{i}\right)$ denotes depth values from the analytical solution for the corresponding spatial coordinates $x_{i}$, and $N$ denotes the number of computational nodes. The resulting values of the mean square error are displayed in Tab. 1 .

Table 1. Mean square error values for the test cases

\begin{tabular}{|c|c|c|c|}
\hline$n\left[\mathrm{~s} / \mathrm{m}^{1 / 3}\right]$ & arithmetic & geometric & harmonic \\
\hline 0.03 & $4.87 \cdot 10^{-7}$ & $8.68 \cdot 10^{-7}$ & $2.56 \cdot 10^{-7}$ \\
\hline 0.031 & $3.56 \cdot 10^{-4}$ & $3.28 \cdot 10^{-4}$ & $3.01 \cdot 10^{-4}$ \\
\hline
\end{tabular}

It can be noticed that the change in Manning's roughness coefficient had a great impact on the solution error. The 3.33\% difference in Manning's roughness coefficient caused the mean square error to increase about a thousand times. However, the errors for each of the solution methods are of the same order. The outcome of the computations is depicted in Fig. 8. The solution is expressed by the relative depth $H_{i} / H\left(x_{i}\right)$ function. The horizontal line at a value of $H_{i} / H\left(x_{i}\right)=1$ denotes the analytical solution.

The roughness coefficient is estimated on the basis of tables with descriptive entries, such as "smooth texture," "corrugated metal," "earth channel - gravelly" etc., to which numerical values of the coefficient are assigned. The choice of the coefficient 


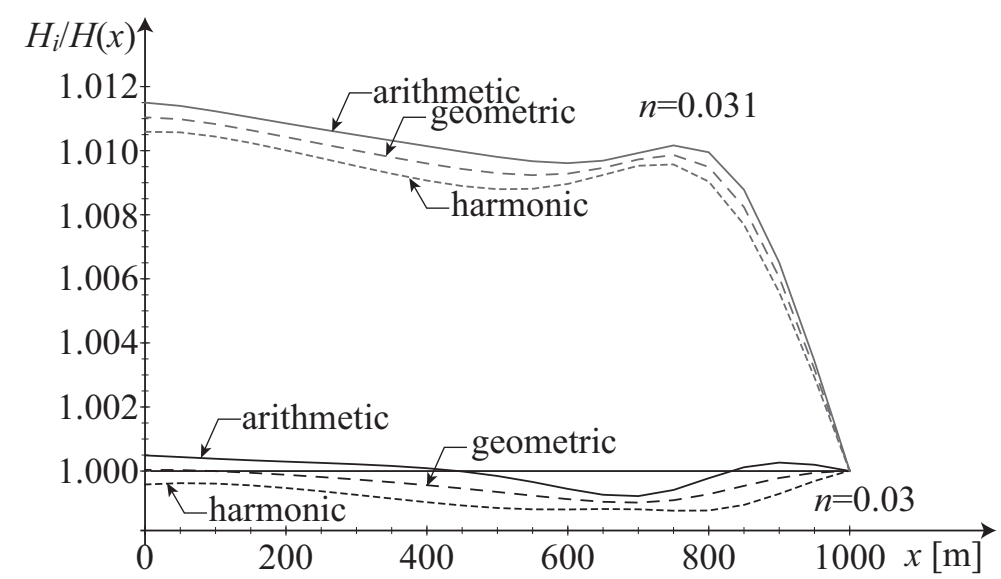

Fig. 8. Comparison of numerical solutions obtained by different numerical schemes and for different values of Manning's roughness coefficient.

is based on a subjective impression of the researcher. Considering the impact of this parameter on the quality of the numerical solution, it is presumably the main source of error in SGVF computations.

\section{Conclusions}

In this paper, averaging methods used in 1D SGVF modelling were examined from the numerical viewpoint. It was shown that the standard step method coincides with the trapezoidal rule, a well-known method for numerical solution of ODEs, and that the introduction of different methods for averaging the energy slope results in the application of different numerical schemes for solving the governing equation.

The numerical methods arising from different types of averaging are instances of the generalized integration rule with respect to the interpolant. Arithmetic mean averaging is equivalent to the generalized integration rule with linear interpolation between computational nodes. Geometric and harmonic averaging is equivalent to hyperbolic interpolation. In the sense of derivation, all the methods have a common origin. However, the trapezoidal rule is a linear method, whereas geometric and harmonic averaging results in non-linear integration methods.

The trapezoidal rule, and instances of the GIR with geometric and harmonic mean averaging were analysed for their basic numerical properties. All methods are consistent of the second order and stable. The trapezoidal rule with arithmetic and geometric averaging is 0 -stable. When the harmonic mean is used, the resulting method is not 0 -stable, and therefore not convergent.

Artichowicz and Mikos-Studnicka (2014) showed that all the methods of averaging the energy slope give almost identical solutions when applied to the SGVF equation. The analyses presented in this paper show that the rule with arithmetic averaging seems to be the best choice for solving the SGVF equation. Although the 
integration rule with geometric averaging has identical basic numerical properties, it is a non-linear method and, as such, may cause various problems in the process of numerically solving a differential equation. For example, the geometric mean in the form discussed here is valid only for non-negative numbers.

Non-linear methods of integrating ODEs are introduced when dealing with specific (usually stiff) differential equations. Then the process of deriving numerical integration schemes is conducted in such a way as to work around issues specific of differential equations considered, or to increase the accuracy of methods applied to a given problem (Fatunla 1982, Lambert and Shaw 1965, Luke et al 1975). The solution of 1D SGVF is not such a case.

The conclusion of this work is that different methods of averaging applied in solving differential equations introduce different numerical schemes and should be avoided if their properties were not formally examined. Such proceeding may introduce serious numerical consequences such as lack of stability or convergence. For this reason, linear integration methods should be used for solving SGVF, as they are very well examined.

Additionally, it should be highlighted that, in the case of 1D SGVF, the main source of error is the estimation of Manning's roughness coefficient. Therefore, changing the numerical integration scheme will usually not increase the accuracy of the solution.

\section{References}

Ascher U. M., Petzold L. R. (1998) Computer methods for Ordinary Differential Equations and Difference-Algebraic Equations, SIAM, Philadelphia.

Artichowicz W., Szymkiewicz R. (2014) Computational issues of solving the 1D steady gradually varied flow equation, J. Hydrol. Hydromech., 62 (3), 226-233, DOI: 10.2478/johh-2014-0031.

Artichowicz, W., Mikos-Studnicka, P. (2014) Comparison of Average Energy Slope Estimation Formulas for One-dimensional Steady Gradually Varied Flow, Archives of Hydro-Engineering and Environmental Mechanics, 61 (3-4), 89-109, DOI: 10.1515/heem-2015-0006

Chanson H. (2004) The hydraulics of open channel flow: an introduction. Second Edition. Elsevier.

Chadderton R. A., Miller A. C. (1980) Friction models for M2 profiles, JAWRA Journal of the American Water Resources Association, 16 (2), 235-242, DOI: 10.1111/j.1752-1688.1980.tb02384.x.

Cunge J. A., Holly F. M., Verwey A. (1979) Practical aspects of computational river hydraulics, Pitman advanced publishing program, Boston, London, Melbourne.

Chow V. T. (1959) Open-channel hydraulics, McGraw-Hill / Kogakusha Company LTD, Tokyo.

Fatunla S. O. (1982) Nonlinear multistep methods for initial value problems, Computers \& Mathematics with Applications, 8 (3), 231-239, DOI: 10.1016/0898-1221(82)90046-3.

French R. H. (1985) Open Channel Hydraulics, McGraw-Hill, New York.

Gustafsson B. (2011) Fundamentals of Scientific Computing, Springer-Verlag, Berlin, Heidelberg.

Gąsiorowski D. (2013) Impact of diffusion coefficient averaging on solution accuracy of the 2D nonlinear diffusive wave equation for floodplain inundation, Journal of Hydrology, 517, 923-935, DOI:10.1016/j.jhydrol.2014.06.039.

Hairer E., Wanner G. (2010) Solving Ordinary Differential Equations II. Stiff and Differential-Algebraic Problems, Second Revised Edition, Springer, Berlin, Heidelberg. 
Kincaid D., Cheney W. (2002) Numerical Analysis, Wydawnictwa Naukowo-Techniczne, Warszawa (in Polish).

Lambert J. D., Shaw B. (1965) A Method for Numerical Solution of $y^{\prime}=f(x, y)$ Based on a Self-Adjusting Non-Polynomial Interpolant, Math. Comp., 20, 11-20, DOI: 10.1090/S0025-5718-1966-0189252-1.

Laurenson E. M. (1986) Friction Slope Averaging in Backwater Calculations, J. Hydraul. Eng., 112 (12), 1151-1163.

LeVeque R. J. (2007) Finite Difference Methods for Ordinary and Partial Differential Equations, SIAM, DOI:10.1137/1.9780898717839.

Luke Y. L., Fair W., Wimp J. (1975) Predictor-corrector formulas based on rational interpolants., Computers \& Mathematics with Applications, 1 (1), 3-12, DOI: 10.1016/0898-1221(75)90003-6.

MacDonald I., Baines M. J., Nichols N. K., Samuels P. G. (1997) Analytic Benchmark Solutions for Open-Channel Flows, J. Hydraul. Eng., 123 (11), 1041-1045.

Szymkiewicz R. (2010) Numerical modeling in open channel hydraulics, Springer.

US Army Corps of Engineers (2010) HEC-RAS hydraulic reference. 